\title{
Application of shear wave elastography and median nerve cross-section area in the diagnosis and staging of carpal tunnel syndrome: a case-control study
}

\author{
Afshin Mohammadi ${ }^{1 A, D, E}$, Ahmadreza Afshar ${ }^{2 A, B, F,}$, Mohammad Mirza-Aghazadeh-Attari ${ }^{3 C, D, E, F}$, \\ Seyed Arman Seyed Mokhtari ${ }^{40, E}$
}

'Department of Radiology, Urmia University of Medical Sciences, Iran ${ }^{2}$ Department of Orthopedics, Urmia University of Medical Sciences, Iran ${ }^{3}$ Department of Radiology, Tabriz University of Medical Sciences, Tabriz, Iran

${ }^{4}$ Students Research Committee, Urmia University of Medical Sciences, Iran

\section{Abstract}

\begin{abstract}
Purpose: Imaging is gaining a more prominent role in the diagnosis of carpal tunnel syndrome (CTS), especially ultrasonography. Shear wave elastography (SWE) is a novel qualitative method to study mechanical changes in tissue. In this study, we aim to assess the role of SWE in diagnosing and staining of the disease.

Material and methods: A total of 124 wrists were included in the study. Seventy wrists had CTS, and 54 were included as the control group. All of the wrists with CTS had staging done with nerve conduction study. All patients underwent ultrasonography by an expert radiologist and had the SWE and cross-section of the median nerve determined. These values were compared among the 2 groups and different stages of CTS. The receiver operating characteristic curve was utilized to assess the diagnostic role of each of the variables.
\end{abstract}

Results: Cross-section area (CSA) and SWE were significantly different between the 2 groups $(p=0.0001)$. CSA was also significantly different among various stages of CTS. SWE was not significantly different among moderate and severe stages of CTS. Both of the variables had a good ability to distinguish mild CTS from healthy wrists $(p=0.0001)$.

Conclusion: SWE can be used in diagnosing CTS and in the staging of the disease.

Key words: shear wave elastography, ultrasonography, median nerve, carpal tunnel syndrome.

\section{Introduction}

Carpal tunnel syndrome (CTS) is characterized by anatomical changes in the median nerve, which leads to the compromise of normal physiological function [1]. The median nerve passes the canal alongside 9 tendons of the flexor muscles, with the flexor retinaculum doming them under the carpal bones. Increased pressures in the carpal tunnel (above the average $2-10 \mathrm{mmHg}$ ) can cause traction- and compression-related alterations in the micro- scopic nerve anatomy [2]. Studies show that the factors mentioned above cause an abnormal decline in the nerve microcirculation, which ultimately leads to oedema and hypoxia of the nerve and causes myelin damage, which interferes with normal signal transduction [3].

Currently, the most utilized method of diagnosis is physical examination combined with secondary diagnostic modalities, such as nerve conduction studies performed alongside electromyography, and imaging methods, such as ultrasound and magnetic resonance imaging [4].

Correspondence address:

Mohammad Mirza-Aghazadeh-Attari, Department of Radiology, Tabriz University of Medical Sciences, Tabriz, Iran, e-mail: m.aghazadeh75@yahoo.com

Authors' contribution:

A Study design · B Data collection · C Statistical analysis · D Data interpretation - E Manuscript preparation · $F$ Literature search · G Funds collection 
Shear wave elastography (SWE) is a recent extension to the diagnostic abilities of ultrasonography, which enables quantitative measurements of the elasticity of tissue. A mechanical study of the median nerve by strain elastography has shown mixed results in assessing carpal tunnel ligament stiffness, the correlation between median nerve is and strain, the pressure of the carpal tunnel, and the elasticity of the nerve [5]. Application of a quantitative method may further the diagnostic implications of ultrasonography in the carpal tunnel, and may even be used as a follow-up marker in subjects.

Considering all of the mentioned points above and the potential benefit of quantitative data in the management and follow-up of patients, the aim of this study is the quantitative measurement of median nerve (MN) stiffness by SWE, in conjunction with cross-section area (CSA) of the median nerve to determine whether it can be useful in the diagnosis and staging of CTS.

\section{Material and methods}

\section{Patients}

The present study was conducted on 124 consecutive subjects presenting to a tertiary medical centre with subspecialized out-patient clinics. The study group was selected from patients with a definite diagnosis of CTS, which was approved by a nerve conduction study and physical examination by an orthopaedic surgeon subspecialized in hand surgery. The control group was selected from healthy volunteers. Exclusion criteria for both groups of subjects included those with prior wrist injury or surgery in the volar aspect of the hand, anatomical anomalies from birth, diabetes mellitus, other neuropathies, a mass lesion in the carpal tunnel, and those refusing to participate in the study.

\section{Nerve conduction study}

Nerve conduction study (NCS) was performed by an electrophysiologist with at least 20 years of experience, according to the guidelines and recommendations issued by the American Association of Neuromuscular and Electrodiagnostic Medicine (AANEM) [6]. Patients were classified as mild, moderate, and severe CTS based on the measurement of the median nerve conduction velocity $[7,8]$. The electrophysiologist was blinded to the clinical characteristics of patients before performing the NCS.

\section{Ultrasonographic imaging}

Ultrasonographic (US) examinations of the hands of the patients and healthy subjects were performed by a radiologist with more than 13 years of experience in musculoskeletal imaging. US imaging was performed using an AixPlorer scanner (AixPlorer Ultimate, Supersonic Imaging, Paris, France) equipped with high-frequency broadband (5-18 MHz) linear array transducer (SL 18-5). The radiologist was blinded to the NCS test results and the clinical characteristics of the patients. US examination was performed within 7 days of the NCS, and the CSA of the median nerve was measured at the level tunnel inlet (level of pisiform bone) (Figure 1).

\section{Protocol of shear wave elastography}

Subjects were seated facing the radiologist with arms extended forward and resting on a hard, flat-surface hand rest, with wrists extended 30 to 45 degrees, forearms supinated, and fingers semi-flexed. The axial B-mode images of the nerve were taken at the level of the pisiform and scaphoid bones (tunnel inlet level). The probe was oriented so that the US beam remained perpendicular to the median nerve, and the wrist was maintained in a supine position with no external pressure applied during the supersonic SWE (SSWE) examination. So that any unwanted and confounder alterations in CSA remained limited, SSWE was performed in dual mode (i.e., elastograms displayed alongside greyscale sonograms in real-time). The radiologist chose the best static SSWE display images in a rectangular region of interest (ROI), which was

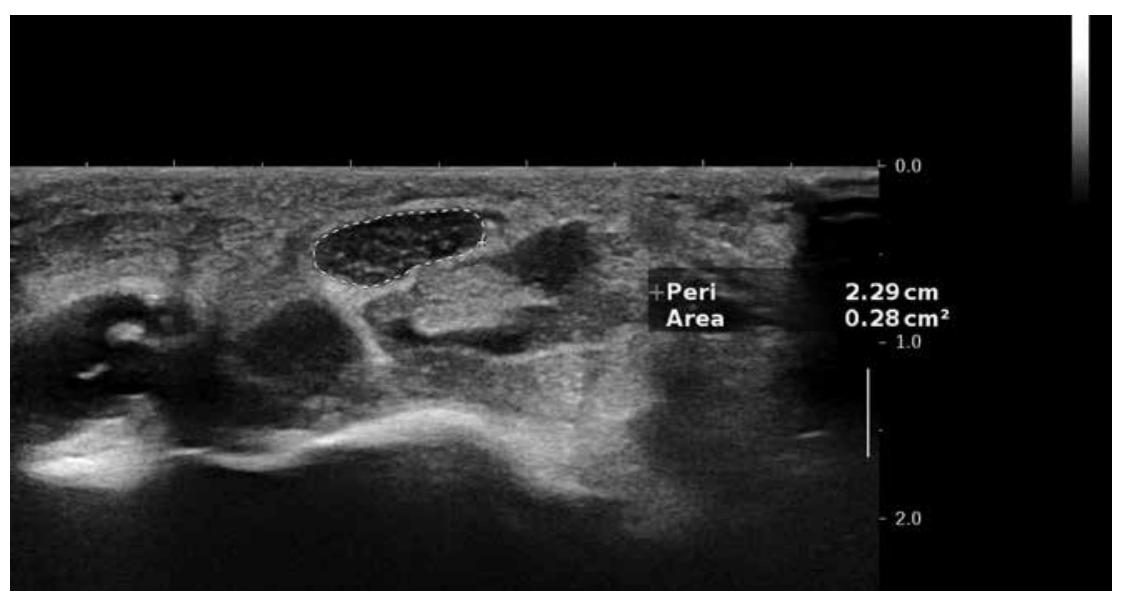

Figure 1. Transverse ultrasonogram was used to evaluate the cross-sectional area of the median nerve at the carpal tunnel in a patient with severe carpal tunnel syndrome 


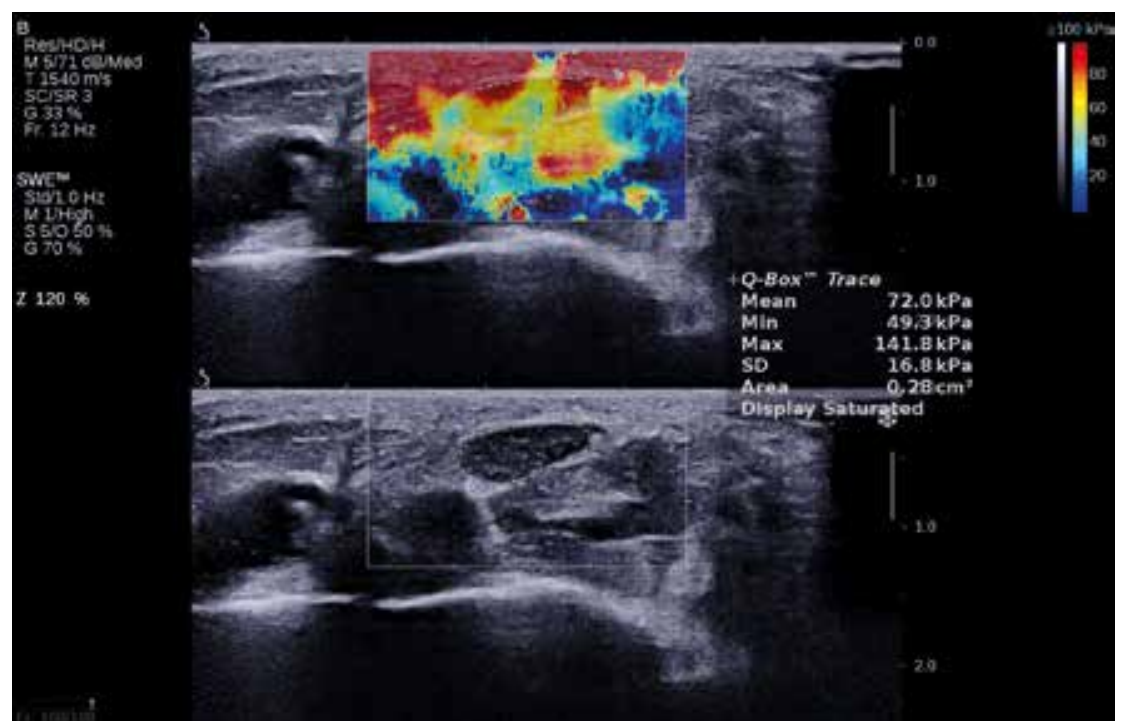

Figure 2. Transverse ultrasound shear wave elastogram was used to evaluate median nerve stiffness measurement in a patient with severe carpal tunnel syndrome

fixed in all the measurements for each subject. Median nerve stiffness was measured by manual curser around the median nerve (Figure 2). The manual ROI around the median nerve in the quantitative analysis provided the CSA of the ROI, and the maximum, minimum, standard deviation of median nerve stiffness in kilopascals $(\mathrm{kPa})$.

\section{Statistical analysis}

Statistical analysis was done using SPSS (version 23.1.0, Chicago, Il, Us). Quantitative data were presented as mean \pm standard deviation. The mean values of CSA and SWE were compared between the 2 groups using an independent $t$-test. One-way ANOVA, coupled with Fisher's least significant difference (LSD) post hoc test, was used to compare CSA and SWE values among various grades of CTS based on NCS. Roc curve analysis was performed for the diagnostic abilities of SWV and CSA, the area under the curve was calculated, and the Youden's index was defined for each variable.

\section{Ethical considerations}

The present study was conducted after gaining ethical approval by the local ethics board of the university where it was performed. All patients had signed written informed consent forms before inclusion. This study was in agreement with the latest version of the Helsinki declaration.

\section{Results}

A total of 124 hands with CTS were included in our study. The mean age of those being included was $50.8 \pm 10.2$ years (range: 19-77 years). Seventy hands were previously diagnosed with CTS, and 54 hands were included as the control group. Of those diagnosed with CTS, NCS revealed that 18 had mild, 26 had moderate, and 26 had severe CTS.
The mean CSA of the median nerve was $0.12 \pm 0.04 \mathrm{~cm}^{2}$ in the study group and $0.05 \pm 0.015 \mathrm{~cm}^{2}$ in the control group. The difference between the 2 groups was statistically significant $(p=0.0001)$.

The median nerve stiffness was $56.55 \pm 20.5 \mathrm{kPa}$ in the patient group and $23.71 \pm 10.3 \mathrm{kPa}$ in the control group, with the difference being statistically significant $(p=0.0001)$.

CSA and median nerve stiffness values were compared between the 4 groups of patients identified by NCS (healthy hands and mild, moderate, and severe CPS). Analysis of variance revealed that both factors were significantly different among the groups. CSA was significantly different when comparing any of the 4 groups to each other, while median nerve stiffness did not show a statistically significant difference when comparing severe CTS to moderate CPS.

Figure 3 demonstrates the linear regression model between CSA and SWE values $(p=0.001)$. The regression coefficient was 0.607 .

The area under the ROC curve for median nerve stiffness and CSA was 0.936 (0.895-0.978) and 0.960 (0.929$0.990)$, respectively. Each of the variables showed an excellent diagnostic profile based on ROC curves $(p=0.0001)$. The ROC curve is shown in Figure 4. A CSA more of than 0.07 had a sensitivity of 88.8 and specificity of 88.4 in diagnosing CTS.

A median nerve stiffness of more than $33.9 \mathrm{kPa}$ had a sensitivity of $90.28 \%$ and specificity of $88.4 \%$ in diagnosing CTS.

The diagnostic profile of SWE and CSA in differentiating mild cases from normal subjects was also assessed. The area under the curve was $0.87(0.782-0.957)$ and 0.907 (0.826-0.988) for SWE and CSA, respectively $(p=0.0001)$. The corresponding ROC curve is shown in Figure 5.

Binominal regression of the 2 variables (SWE and CSA) was used to assess their effects on the likelihood 


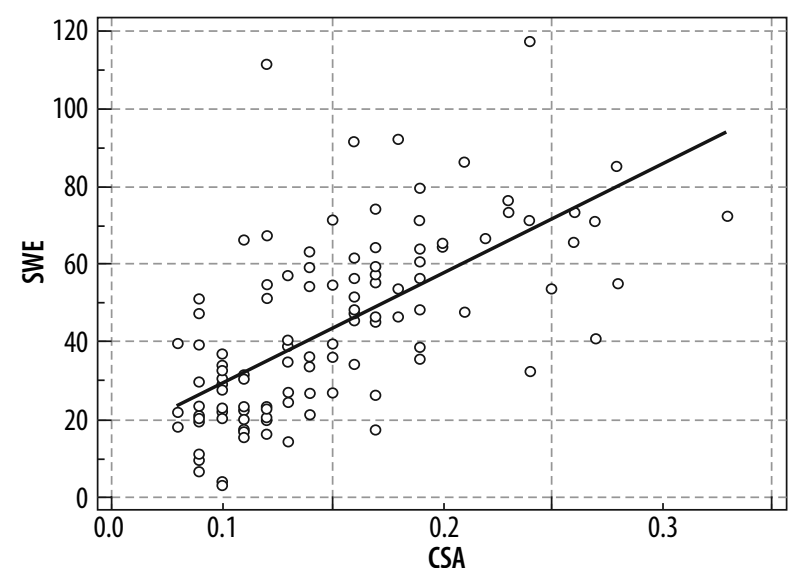

Figure 3. Scatter plot and the trend line for shear wave elastogram (SWE) and cross-section area (CSA). Regression coefficient equalled 0.607

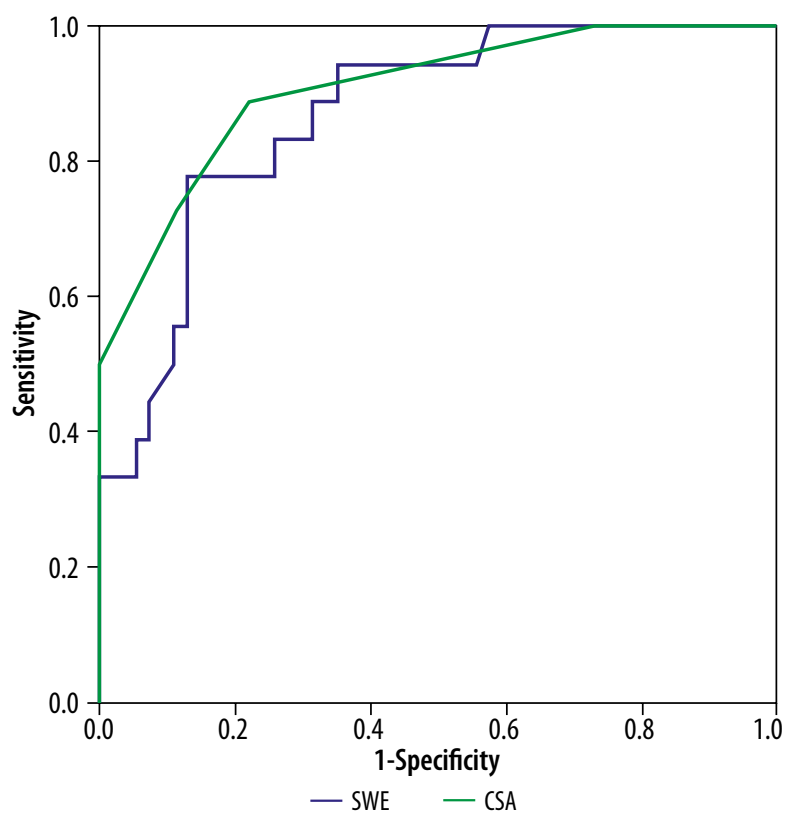

Figure 5. ROC curve presenting the diagnostic profile of cross-section area (CSA) and shear wave elastogram (SWE) in differentiating mild carpal tunnel syndrome from normal wrists

of participants having CTS. The results revealed a significant contribution of each of the 2 variables ( $p=0.0001$ for CSA and $p=0.001$ for SWE) and an overall statistically significant model $\left(\chi^{2}[2]=128.9, p=0.0001\right)$. The model correctly predicted $87 \%$ of the variance in CTS among participants (Nagelkerke's $R^{2}$ ).

The overall sensitivity of the model was $95.2 \%$ and specificity was $95.8 \%$.

An ROC curve based on this model yielded an area under the curve of 0.977 (0.951-1). Figure 6 depicts the associating ROC curve.

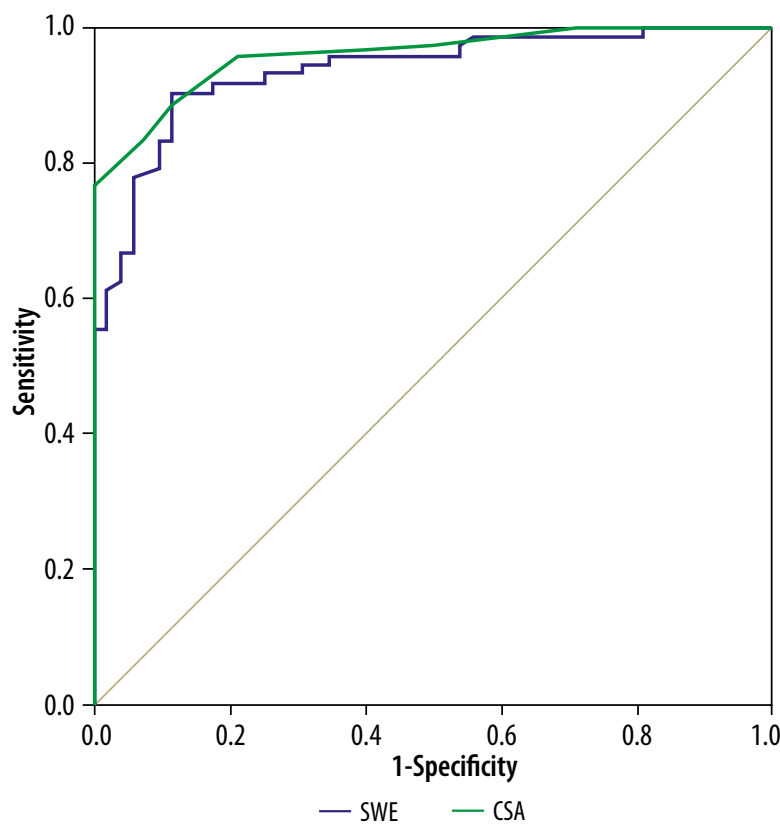

Figure 4. ROC curve of the diagnostic profile of shear wave elastogram (SWE) and cross-section area (CSA) in differentiating carpal tunnel syndrome cases (all stages included) and healthy wrists

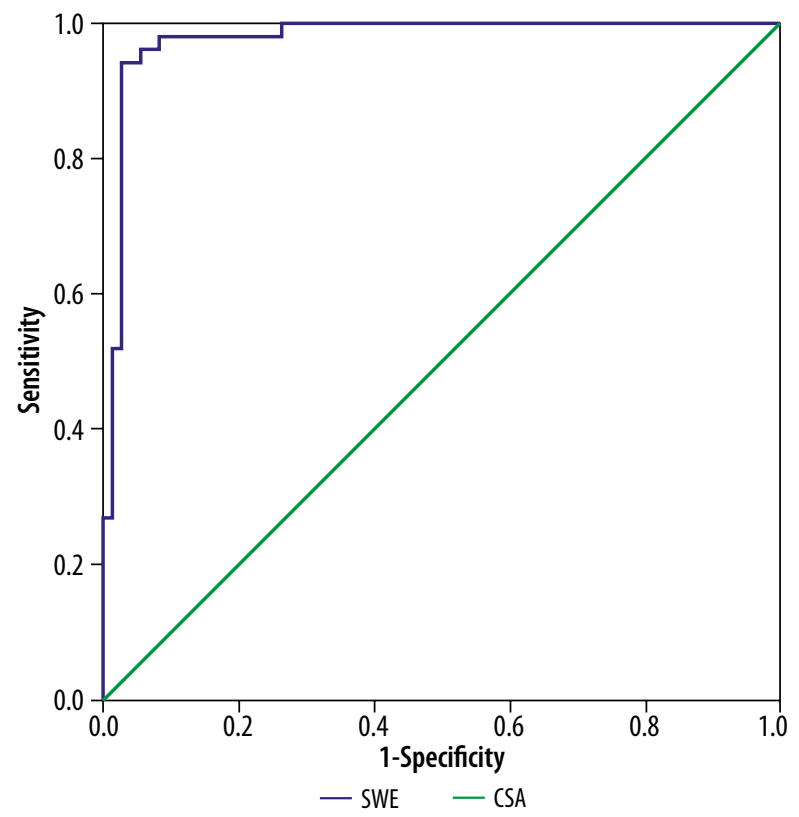

Figure 6. ROC curve of the bivariate model using shear wave elastogram (SWE) and cross-section area (CSA) as entry predictors (AUC $=0.97,0.951-1.0)$

\section{Discussion}

In the present study, we evaluated the diagnostic value of SWE and the cross-section of the median nerve in CTS. Our results demonstrate a favourable diagnostic profile for both of the parameters, and we also show that these variables can be used in classifying CTS based on severity.

Ultrasonography has been used in the diagnosis of peripheral neuropathies such as CTS for some time. Conventionally, strain elastography has been considered. In contrast to SWE, strain elastography is a qualitative 
method, in which the operator exerts manual force to tissue via a transducer that senses the degree of deformation compared to the surrounding tissue. This method is highly operator dependent, and the technical specifications of the imaging, such as the size of the region of interest, may hinder the reproducibility of any results. Furthermore, interpretation of qualitative data may prove confusing and inaccurate, especially in the follow-up of patients undergoing conservative treatment. Amid these limitations, clinical studies show contradictory results. He et al. showed that SWE values were significantly different among the median and tibial nerve of patients with diabetic neuropathy and the control group. It also had a higher area under the curve compared to CSA $(0.927$ compared to 0.798 in the tibial nerve) [9]. Similar results were seen in ulnar neuropathy associated with diabetes and ulnar tunnel syndrome $[10,11]$.

Martin et al. did not find a significant difference in strain values among 17 CTS patients and 26 control subjects [12], while Emara et al. reported a significant difference not only in CTS patients and control cases but also between different severities of CTS [13]. A systemic review by Lin et al. reported a standardized mean difference of 1.307 (95\% CI: 0.876-1.737, $\mathrm{I}^{2}=78.9 \%$ ) for the strain ratio in CTS patients vs. healthy subjects. Although the study did not yield a significant result, the authors concluded that there was potential for further investigation of the use of strain elastography because the studies had somewhat different imaging techniques, and the results may have been confounded by them [14]. However, a study by Yoshii et al. was able to show that strain elastography of the median nerve could be a prognostic factor in patients undergoing median nerve release due to CPS. They suggest that the area under the curve for the pressure, strain, pressure-strain ratio, motor latency, sensory latency, and area were $0.689,0.773,0.811,0.668$, 0.637 , and 0.562 , respectively, which shows a fair diagnostic profile in most cases, with the strain ratio having the most AUC [15].

SWE is not studied as thoroughly as strain elastography. Zhang et al. studied 72 wrists with CPS and 46 healthy wrists and found that the SWV was 5.241 $\pm 0.545 \mathrm{~m} / \mathrm{s}$ in CPS wrists and $3.248 \pm 0.410 \mathrm{M} / \mathrm{S}$ in the control group, with the difference being significant. The same was also true for CSA. Interestingly, they were able to show that the SWV values were significantly different between patients with mild or moderate CTS and severe CTS [16].

Ardakani et al. studied the application of SWE, morphometry, and artificial intelligence techniques in the diagnosis of CTS. They found that various parameters of elastographic imaging, including the elasticity mean, the standard deviation of elasticity, blue index, red index, and softness index were significantly different among control subjects and CTS patients. They incorporated the data generated by elastographic imaging and morphometry to 2 models of traditional machine learning and deep learning models. In the conventional model, an AUC of 0.943 , sensitivity of $90 \%$, specificity of $95 \%$, and accuracy of $92.50 \%$ were achieved. The deep learning model yielded an even better response: the AUC, sensitivity, specificity, and accuracy were $0.980,95.00 \%, 100 \%$, and $97.50 \%$, respectively [17].

In a recent study by Wee et al., 47 wrists with CTS were compared to 25 wrists from control patients. The CSA of CTS wrists was $11.5 \pm 3.4 \mathrm{~mm}^{2}$ compared to $7.3 \pm 1.9$ in normal wrists $(p=0.001)$. The authors also calculated the wrist to forearm the CSA ratio of the median nerve and found that it was significantly different in CTS patients. Unlike previous studies, the CSA and CSA ratio were not significantly different among different severities of CTS. The authors suggested that the optimal median nerve CSA cut-off value for the diagnosis was 1.45 [18].

In this study, we showed that a significant correlation existed between SWE and CSA. This can be justified by the fact that as the nerve increases in diameter due to oedema and fibrous tissue formation, the elasticity decreases. CSA is an anatomical factor, and the addition of SWE or other markers of nerve physiology may be useful in more accurate analysis of imaging findings, differentiating conditions that only affect 1 of the variables, or interpreting both of the variables in light of changes made to the other one. Although our results show a favourable diagnostic profile for SWE in CTS, our results should be interpreted with consideration of the limitations of our study. Our study was conducted on patients presenting to a single medical centre. Thus, the diversity of our patients may not reflect that of our general population. Studies that are performed on multiple centres and include a wide range of patients with pre-existing conditions such as diabetes, peripheral neuronal disease, and athletes or others with variations in the components of the musculoskeletal system compared to the general population may reveal the true diagnostic accuracy of SWE in CTS, as well as other peripheral neuropathies.

\section{Conclusions}

In the present study, we showed that CSA and SWE were significantly different between CTS wrists and the control group $(p=0.0001)$. CSA was also significantly different among various stages of CTS and could be used as a staging variable. SWE was not significantly different among moderate and severe stages of CTS. Both of the variables had a good ability to distinguish mild CTS from healthy wrists $(p=0.0001)$. There was a significant correlation between CSA and SWE, which can be further utilized in image analysis and diagnostic approaches.

\section{Conflicts of interest}

The authors report no conflict of interest. 


\section{References}

1. Aroori S, Spence RA. Carpal tunnel syndrome. Ulster Med J 2008; 77: 6-17.

2. Soubeyrand M, Melhem R, Protais M, Artuso M, Crézé M. Anatomy of the median nerve and its clinical applications. Hand Surg Rehabil 2020; 39: 2-18.

3. Aboonq MS. Pathophysiology of carpal tunnel syndrome. Neurosciences 2015; 20: 4-9.

4. Ghasemi-Rad M, Nosair E, Vegh A, et al. A handy review of carpal tunnel syndrome: from anatomy to diagnosis and treatment. World J Radiol 2014; 6: 284

5. Schrier VJ, Lin J, Gregory A, et al. Shear wave elastography of the median nerve: a mechanical study. Muscle Nerve 2020; 61: 826-833.

6. Fuglsang-Frederiksen A, Pugdahl K. Current status on electrodiagnostic standards and guidelines in neuromuscular disorders. Clin Neurophysiol 2011; 122: 440-455.

7. Roll SC, Volz KR, Fahy CM, Evans KD. Carpal tunnel syndrome severity staging using sonographic and clinical measures. Muscle Nerve 2015; 51: 838-845.

8. Kleindienst A, Hamm B, Lanksch WR. Carpal tunnel syndrome: staging of median nerve compression by MR imaging. J Magn Reson Imaging 1998; 8: 1119-1125.

9. He Y, Xiang X, Zhu BH, Qiu L. Shear wave elastography evaluation of the median and tibial nerve in diabetic peripheral neuropathy. Quant Imag Med Surg 2019; 9: 273.

10. Paluch Ł, Noszczyk B, Nitek Ż, Walecki J, Osiak K, Pietruski P. Shear-wave elastography: a new potential method to diagnose ulnar neuropathy at the elbow. Eur Radiol 2018; 28: 4932-4939.
11. Noszczyk B, Walecki J, Osiak K, Kiciński M, Pietruski P. Shear-wave elastography in the diagnosis of ulnar tunnel syndrome. J Plast Reconstr Aesthet Surg 2018; 71: 1593-1599.

12. Martin MJ, Cartwright MS. A pilot study of strain elastography in the diagnosis of carpal tunnel syndrome. J Clin Neurophysiol 2017; 34: 114-118.

13. Emara DM, El Shafei MM, Naby HMA. Utility of ultrasound elastography in a cohort of patients with idiopathic carpal tunnel syndrome. Egypt J Radiol Nucl Med 2018; 49: 408-414.

14. Lin CP, Chen J, Chang KV, Wu WT, Özçakar L. Utility of ultrasound elastography in evaluation of carpal tunnel syndrome: a systematic review and meta-analysis. Ultrasound Med Biol 2019; 45: 28552865.

15. Yoshii Y, Tung WL, Yuine H, Ishii T. Postoperative diagnostic potentials of median nerve strain and applied pressure measurement after carpal tunnel release. BMC Musculoskelet Disord 2020; 21: 22.

16. Zhang C, Li M, Jiang J, et al. Diagnostic value of virtual touch tissue imaging quantification for evaluating median nerve stiffness in carpal tunnel syndrome. J Ultrasound Med 2017; 36: 1783-1791.

17. Ardakani AA, Afshar A, Bhatt S, et al. Diagnosis of carpal tunnel syndrome: a comparative study of shear wave elastography, morphometry, and artificial intelligence techniques. Pattern Recogn Lett 2020; 133: 77-85.

18. Wee TC, Simon NG. Shearwave elastography in the differentiation of carpal tunnel syndrome severity. PM\&R 2020. DOI: https://doi. org/10.1002/pmrj.12334. 